\title{
Operation method of allowing variation interval of downstream water level in the middle route of South-to-North Water Diversion Project
}

\author{
Wu Yongyan ${ }^{1, a}$, Huang Huiyong ${ }^{1}$, Li Qi $^{1}$, Yan Yibo ${ }^{1}$ \\ ${ }^{1}$ Changjiang Institute of Survey, Planning, Design and Research, Planning department, Wuhan, Hubei 430010, China
}

\begin{abstract}
To realize demand-oriented operations in the middle route of South-to-North Water Diversion Project, this paper proposed a pool operation method that a variation interval of water level at the upstream of the check gate is allowed. In this method, water users' demand for flow changes can be satisfied as much as possible. Accordingly, downstream water depth is kept to fluctuate within a certain range, which is determined considering the canal safety, the operation frequency of check gates and the pool delay. The method is tested using the self-developed simulation platform for the middle route of South-to-North Water Diversion Project, which is established based on the Saint-Venant equations and programmed with the language Fortran. Results show that the flow changes demanded by water users, either increase or decrease, can be achieved effectively, while the water level variations can meet the restrictions for canal operation safety, and the frequency of gate adjustment is practically acceptable as well. Therefore, the operation method of allowing variation interval of downstream water level is suitable in the middle route of South-to-North Water Diversion Project at the current stage.
\end{abstract}

\section{Introduction}

There are several methods of pool operation for longdistance diversion project to convey water to downstream water users. The method of pool operation usually determines a canal's recovery characteristics, namely, the speed and manner in which the canal recovers to a new steady state after a flow change ${ }^{[1]}$. The main methods of pool operation are constant downstream depth method, constant upstream depth method, constant volume method and controlled volume method. Among the four methods, the constant downstream depth method is the one mostly used in water diversion projects home and abroad for its simplicity and reliability. The volume controlled method has been successfully used in the Central Arizona water diversion project and the water transfer project from Yellow River to Qingdao, but it is comparatively less used in engineering projects for its high requirements for automation facilities.

The middle route of South-to-North water diversion project adopts the constant downstream depth method at the preliminary design stage. However, this method cannot fully meet the requirements of automation operation currently, since some new working conditions have occurred, of which the most significant change is that the demand-oriented delivery strategy is employed instead of the supply-oriented strategy for water users' benefits. It is known that if the downstream water depth is maintained constant, the inflow must overcompensate for the outflow change. Therefore, changes in demand should be predicted, so that the inflow change can be made in advance of the outflow change. When water users change their flows provisionally, the check gates may be regulated frequently, and the system stability would be rather poor.

Several researches have been done to overcome this deficiency from the automatic control aspect, but they mainly focus on improving control algorithms. Rodellar et al. ${ }^{[2]}$ used the predictive control strategy to formulate a control law which can manipulate the upstream gate to make the downstream discharge meet the demanded profile. To achieve real-time operation, they used only upstream and downstream water level measurements as feedback information, and ignored discharge measurements to deal with unknown disturbances in the reach caused by unmeasured water offtakes. Liu et $\mathrm{a}^{[3]}$ adopted a sufficient large recovery time in their control algorithm to cope with rapid variations of water demand and to achieve a stable control of the system. Unfortunately, the above two controllers were only used to a single canal reach for test. Barros et $\mathrm{al}^{[4]}$ developed a modified EL-FLO plus RESET control algorithm to translate the reading of the downstream water level to its corresponding upstream gate opening almost instantaneously. They proposed an optimization model to assist in the controller design for a more efficient canal control, and introduced two additional parameters - the error allowance and the adjustment factor to ensure a smooth upstream gate operation at the same time. Their numerical test, performed on a canal system consisting of

${ }^{a}$ Corresponding author: 1031880619@qq.com 
five pools and eight canal-side turnouts, showed acceptable results, but failed to consider the deadband of the check gates.

In this paper, the authors tried to improve the response of a canal system to water users' demand by proposing an improved method of pool operation, whereby a variation interval of water level at the upstream of the check gate is allowed. In this way, the pool storages can be made full use of to respond to turnout flow changes, planned or unplanned (including peak pumping), large or small, without too many check gates being frequently operated. The main characteristics of this operation method are: (1) the water level fluctuation at the upstream of the check gate is restricted to a certain level; (2) the drawdown rate, namely, the water level declining speed, is limited within a certain value, depending on the concrete lining condition and the drainage condition; (3) the check gates are allowed to be operated simultaneously. The method were applied to the open channel section of the middle route of Southto-North water diversion project, which starts from Taocha headgate and ends at Beijuma check gate. A normal case in which the turnout flow either increases or decreases was considered herein numerically.

\section{Hydrodynamic Simulation Model}

The open channel of the middle route of the South-toNorth water diversion project is mild slope, and as the length is much larger than the cross-section, the flow regulated by check gates can be considered as onedimensional unsteady, gradual flow. Therefore, The SaintVenant equations could be used:

$$
\begin{gathered}
\frac{\partial Z}{\partial t}+\frac{1}{B} \frac{\partial Q}{\partial x}+\frac{q}{B}=0 \\
\frac{\partial Q}{\partial t}+\frac{2 Q}{F} \frac{\partial Q}{\partial x}+g F \frac{\partial Z}{\partial x}-\frac{Q^{2}}{F^{2}} \frac{\partial F}{\partial x}+\frac{g Q^{2}}{F C^{2} R}=0
\end{gathered}
$$

where $Z$ is the water level, $Q$ is the discharge, $F$ is the flow cross-section area, $B$ is the water width, $t$ is the time, $x$ is the distance along the flow direction, $q$ is the lateral inflow per unit length, $g$ is the gravitational acceleration, $R$ is the hydraulic radius, and $C$ is the Chezy coefficient. As the Saint-Venant equations neglect the local losses, the manning's roughness coefficient of open channel should take a somewhat larger value than design.

The continuity equation (1), and the momentum equation (2), describe the flow within each pool between the upstream and downstream check gates. The interior boundary condition is the discharge equation of check gates, which can be describe by the following general forms for orifice flow and weir flow, respectively:

$$
\begin{aligned}
& Q=\sigma m \varepsilon b e \sqrt{2 g h} \\
& Q=\sigma m \varepsilon b e \sqrt{2 g} h^{1.5}
\end{aligned}
$$

Where $\sigma$ is the submergence coefficient, $m$ is the discharge coefficient of the check gate, $\varepsilon$ is the lateral contraction coefficient, $b$ is the upstream channel width at half depth, $e$ is the opening of the check gate, $h$ is the difference in water level upstream and downstream of the check gate. For the transition flow from the orifice flow to the weir flow, the discharge is obtained by linear interpolation.

The hydrodynamic simulation model is solved by the Pressimann four-point implicit finite difference scheme. The canal is discretized by a set of nodes $\left(x_{i}, i=1,2, \ldots, m\right)$. The level of discretization is chosen so that the resulting numerical solution is sufficient to represent the continuous functions $Q(x, t)$ and $Z(x, t)$. After spatial discretization, the two partial differential equations (1) and (2), are replaced by $2 m$ nonlinear ordinary differential equations at each time step of integration. The computation was accomplished by the program language Fortran in this study.

\section{Method of pool operation}

To take full advantage of pool storages, water level at the upstream of the check gate is allowed to fluctuate around the target water level, thereby, the demand of water users for minor flow changes can be satisfied as quickly as possible, and the check gates could avoid needless or frequent operation caused by the pool delay. Specific requirements of water level in this method are:

(1) The water level should not exceed the maximum permissible level to avoid overtopping;

(2) The water level should be above the minimum permissible level to guarantee delivery and safe operation of inverted siphons.

(3) The water level drawdown rate should not exceed a certain level to prevent the lining from damaged. The limit value depends on the concrete lining condition and the drainage condition, which is recommended to be $0.15 \mathrm{~m} / \mathrm{h}$ and $0.3 \mathrm{~m} / \mathrm{d}$ by the Reclamation bureau, and taken as $0.3 \mathrm{~m} / \mathrm{h}$ and $0.7 \mathrm{~m} / \mathrm{d}$ in the water transfer project from Yellow River to Qingdao ${ }^{[5]}$. For the middle route of South-to-North water diversion project, it is suggested to be $0.3 \mathrm{~m} / \mathrm{d}$ in the preliminary design report for safety considerations.

(4) The variation interval of water level at the upstream of the check gate should be set reasonably. A too large variation interval is unfavorable to the water surface stability and open channel safety, while a too small variation interval limits the availability of pool storage and may weaken the effectiveness of this method. In this study, the lower limit is set $0.1 \mathrm{~m}$, and the upper limit is set $0.15 \mathrm{~m}$, according to the operation experience of the middle route of South-to-North water diversion project.

In this pool operation method, several check gates are allowed to be operated simultaneously, and flow changes of water users can be satisfied immediately if only the pool storage and water level conditions permitted. When small temporary flow changes of water demand occur, such us daily peak pumping, the pool storage can be used and only minor or even no adjustments of check gates are needed to be made. When the water level at the upstream of the check gate exceed the set limit, either the upstream check gate or the downstream check gate could be operated, depending on the whole volume balance condition. For example, when the water level at the upstream of the check gate is above the target and beyond the fluctuation allowance, some check gates should be operated to bring the water level back to target. If the downstream pool 
storages are generally in surplus, then the upstream check gates should close; otherwise, the downstream check gates could open. All check gates should be operated gradually and based on the real-time water level, to prevent excessive travel of check gates. However, in case that the water level moves away from the target further, some turning back of the relevant check gates is allowed to ensure canal safety.

\section{Numerical simulation}

The method is applied to the open channel section of the middle route of South-to-North water diversion project, which is 1197.8 kilometers long from Taocha headworks to Beijuma River check gate. The canal system studied consists of 60 pools demarcated by 61 check gates. The pools are named from upstream to downstream successively, from 1 to 60 , and the check gates are labeled accordingly from 0 to 60 , as shown in Fig. 1 .

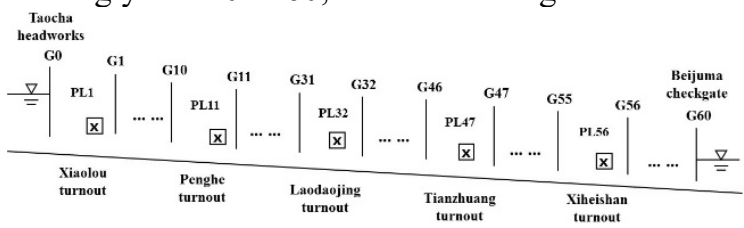

Figure 1. Sketch of the open channel reach of the middle route of South-to-North Water Diversion Project

The canal system comprises 78 canal-side turnouts and different kinds of conveyance structures, such as inverted siphons (including the tunnel crossing Yellow River), aqueducts and culverts. In the simulation model, each canal pool is firstly divided into several subsection by the line where the cross-section change takes place; each subsection is then discretized into several segments by computation nodes. The discretization is uniform (100m) except for the vicinity of check gates, where the discretization is finer. The time step is 60 s to ensure computation accuracy and efficiency. The manning's roughness coefficient is taken as 0.015 , and the water utilization coefficient is assumed as 1 . The operation deadband of check gates, namely, the minimum gate movement, is set as $0.02 \mathrm{~m}$ except that it's $0.05 \mathrm{~m}$ for Taocha headgate, so as to avoid frequent operations of the headworks from management considerations.

The boundary conditions are that the discharge at the end is $30 \mathrm{~m}^{3} / \mathrm{s}$ and the water level at the downstream of Beijuma check gate is $59.9 \mathrm{~m}$. The initial conditions are: (1) the initial water level at the upstream of each check gate (except for Taocha) is $0.1 \mathrm{~m}$ below its respective design water level; (2) the initial turnout flow is $70 \%$ of its respective design discharge, except that it's $3 \mathrm{~m}^{3} / \mathrm{s}$ for Xiaolou turnout. The target water level remains the same as the initial water level. Five water users call for flow changes, as shown in Table.1. Another restraint is that the check gate at the exit of the tunnel crossing Yellow River is manipulated to keep the water level at the entrance of the tunnel around $117.00 \mathrm{~m}$. The simulation period is 100 hours, and the calculation results are output hourly.

\begin{tabular}{cccc}
\multicolumn{3}{c}{ Table 1. Water users' demand for flow changes } \\
\hline Location & $\begin{array}{c}\text { Name of } \\
\text { turnout }\end{array}$ & $\begin{array}{c}\text { Initial } \\
\text { flow }\left(\mathrm{m}^{3} / \mathrm{s}\right)\end{array}$ & $\begin{array}{c}\text { Target } \\
\text { flow }\left(\mathrm{m}^{3} / \mathrm{s}\right)\end{array}$ \\
\hline PL1 & Xiaolou & 3 & 23
\end{tabular}

\begin{tabular}{lccc} 
PL11 & Penghe & 18 & 22 \\
PL32 & Laodaojing & 8.5 & 12 \\
PL47 & Tianzhuang & 45 & 35 \\
PL56 & Xiheishan & 42 & 40 \\
\hline
\end{tabular}

\section{Results}

To satisfy water users' demand for flow changes, the flow at Taocha headworks should increase from $330.70 \mathrm{~m}^{3} / \mathrm{s}$ to $348.20 \mathrm{~m}^{3} / \mathrm{s}$, nearly the design flow of the main canal head. The flow histograms at Xiaolou, Penghe, Laodaojing, Tianzhuang and Xiheishan turnouts are shown in Fig.2, and the opening process of the main relevant check gates are shown in Fig.3. The resulting water level fluctuation at the upstream and downstream of the relevant check gate are shown in Fig.4.

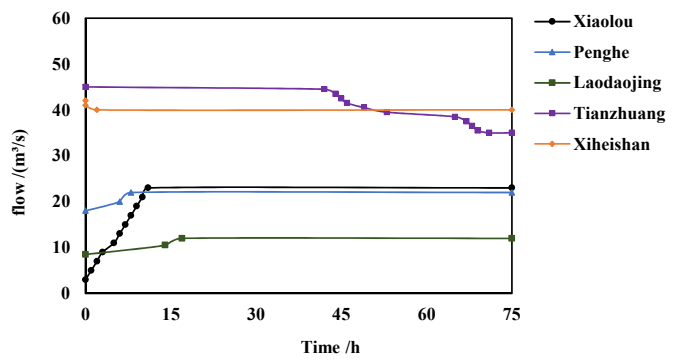

Figure 2. Flow histograms at the turnouts calling for changes

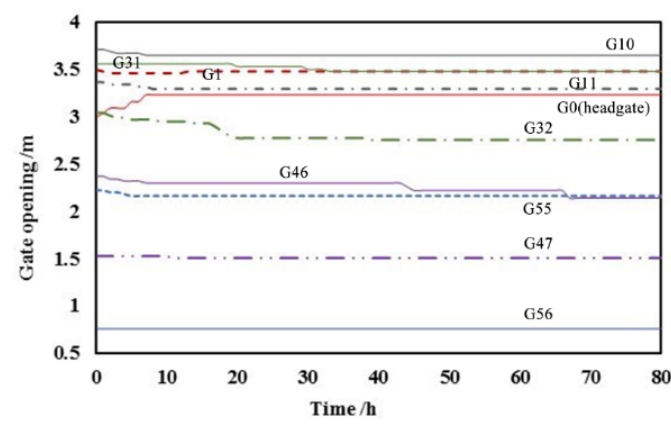

Figure 3. The main relevant gate opening process
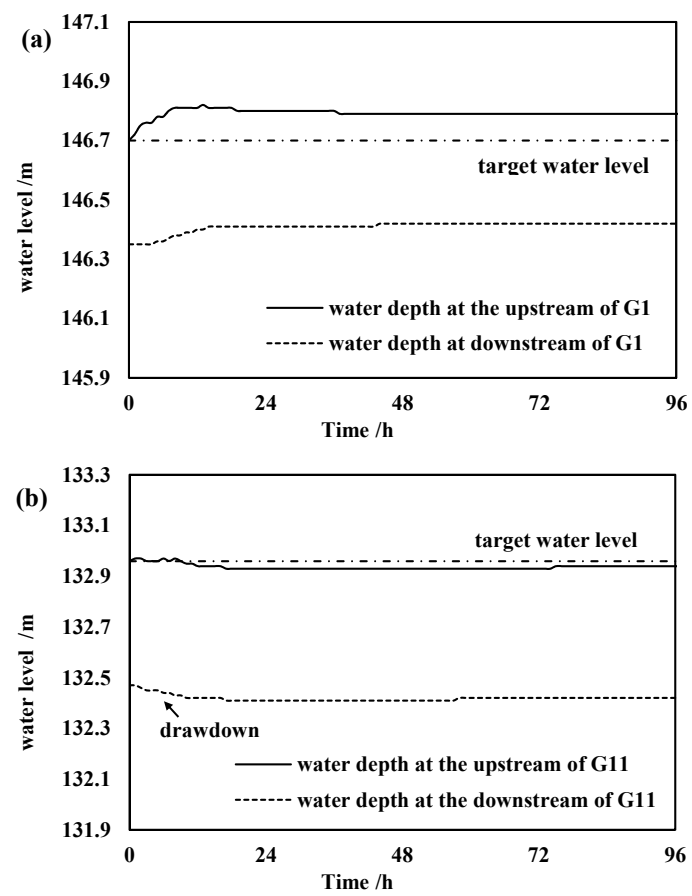

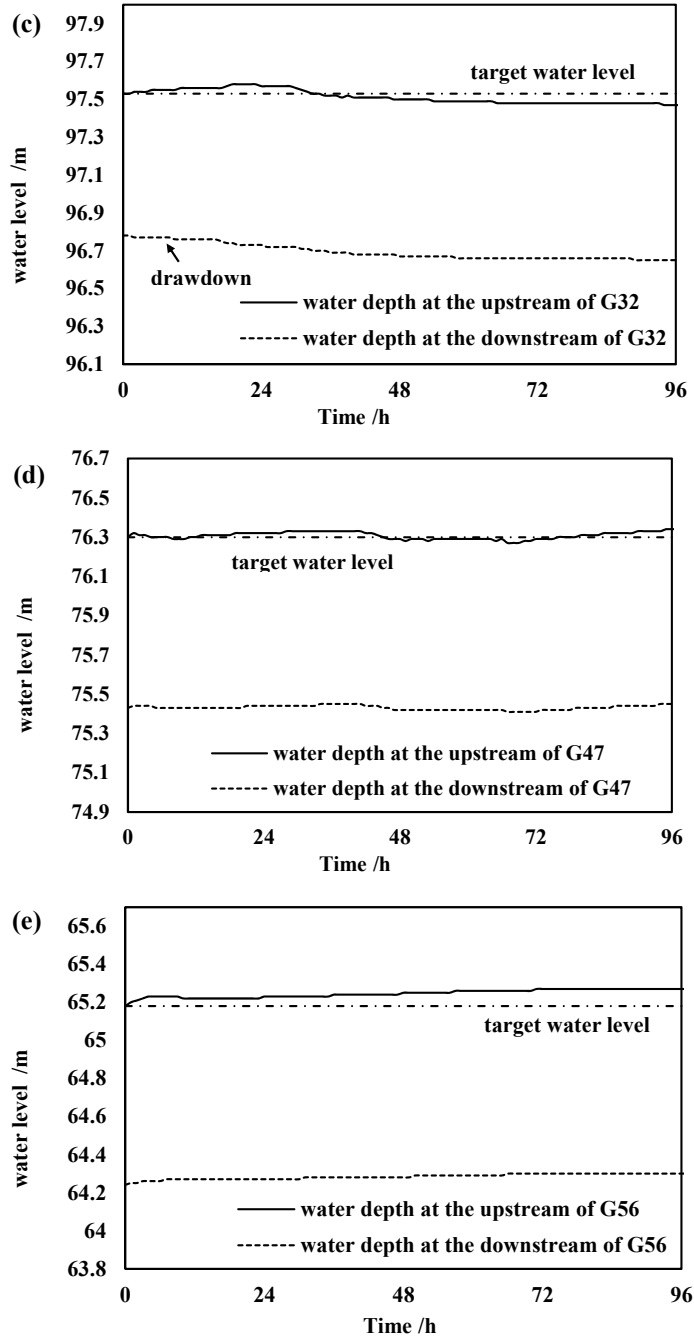

Figure 4. Water level at the upstream and downstream of relevant check gates

Fig.4 shows that during the transition process from the initial state to the target state, the water level at the upstream of each check gate is well kept within the target variation interval, and the daily and hourly drawback rate criteria have also been met, implying that the operation method put forward in this paper functions effectively. The water level deviation at the upstream of Gate 1 is the greatest because the deadband of headgate G0 is larger those of the rest.

The heart of the operation method proposed herein is to meet water users' demand as much as possible. Small flow changes can be immediately made, as long as the pool storage permitting. Therefore, Xiheishan turnout, situated at the lower end of the main canal (PL56), can reduce its flow on demand, as shown in Fig.2. However, Tianzhuang turnout, which is located upstream from Xiheishan turnout and calls for a larger flow change, starts to close after its upstream check gate (G46) closes to a certain extent. Because firstly, large flow decrease can only be accomplished gradually to avoid too rapid drawdown, and secondly, from the pool storage consideration, it is inadvisable to reduce the turnout flow and the outflow of the corresponding pool at nearly the same time, with the latter move is a must to realize the flow decrease of the further downstream turnout. On the other hand, water users' demand for flow increase can be met gradually when the pool inflow is about to increase, or the water level at the upstream of the check gate is going up, as illustrated in Fig.2 and Fig.4. Moreover, Fig.2 shows that the flow of most turnouts exhibits nonlinear changes, since in real-time control, turnout flow changes, pool storage and water level drawdown rate often restrict one another.

After the turnout flow change has been realized, the opening of the downstream check gate of the corresponding pool stays almost the same for long, and the upstream water level deviation of the gate is kept within the variation interval, which demonstrates that the joint action of water level variation interval and deadband of gate operation can effectively prevent frequent adjustments of check gates, a symbol of cost saving and stability. The check gates are operated gradually, therefore, the upstream check gates where flow changes are larger are adjusted more frequently, as shown in Fig.3. Additionally, in this operation method, check gates are operated based on the planned flow changes as well as the real-time measured water level, so as to minimize the effect of pool delay and to avoid excessive gate travel, providing a more stable control of the canal system. However, a little turning back of check gates is allowed for rectification, in case that water level moves away from the target further. Fig. 3 demonstrates that for most check gates, the gate opening changes monotonously, except that Gate 1 firstly closes and then opens, which is because its initial opening and target opening are almost the same, and the operational deadband of headgate $\mathrm{G} 0$ is larger than the rest check gates.

\section{Conclusion}

This paper proposed an improved pool operation method for the demand delivery of long-distance water diversion project, represented by the middle route of South-to-North Water Diversion Project. This method allows a variation interval of water level at the upstream of the check gate, so as to meet water users' demand for flow changes as much as possible, and to avoid frequent adjustments of check gates, thus improving stability of the system. The method proposed here is formulated based on the balance of flow and adopts a real-time control concept, thus abandoning the complicated parameter determination process and presenting stronger applicability. The flow change process is made based on water users' plan as well as the measured water level, and the check gates are operated gradually considering the then pool storage and the water level changing tendency. These strategies can prevent too rapid flow and water level changes, avoid excessive gate travel and minimize the effect of pool delay, ensuring safe and effective canal operation. In addition, some turning back of check gates is allowed for rectification, offering greater flexibility and reliability of the control system. Numerical simulation test has been performed on the open channel reach of the middle route of South-to-North Water Diversion Project (from headgate Taocha to Beijuma check gate), using the self-developed simulation platform programmed with language Fortran. Results show that the flow changes can be realized as far 
as possible, while the water level is kept within the variation interval and meet all the restrictions. Combined with the given deadband of gate operation, check gates are adjusted to the target state without frequent or oscillating operation.

In further study, more research could be done to discuss whether different variation intervals of water level or deadband values of gate operation could provide better performance. Additionally, observation data and manipulation records could be collected to validate the operation method herein, or putting it into trial on practical open channel for demonstration if possible.

\section{Acknowledgement}

This study is sponsored by National Key Research and Development Program of China (Grant No.2016YFC0401810), and Independent Research Project of C.I.S.P.D.R. (CX2017Z04)

\section{References}

1. Y.Y. Wu, H.Y. Huang, Y.B. Yan, et al. Operation method of phase I works of middle route of Southto-North Water Diversion Project. Yangtze River. 2018,49(13): 65-69.

2. J. Rodellar, M. Gomez, L. Bonet. Control method for on-demand operation of open-channel flow. Journal of Irrigation and Drainage Engineering. 1993, 119(2): 225-241.

3. F.B. Liu, J. Feyen, J. Berlamont. Downstream control of multireach canal systems. Journal of Irrigation and Drainage Engineering. 1995, 121(2):179-190.

4. M.T.L. Barros, S.L. Yang, B.P.F. Braga, et al. Optimal design for automatic control of on-demand canal systems. Journal of Infrastructure Systems. 1997,3(2): 59-67.

5. Y. Zhang, J.G. Ma, J.R. Wang, et al. Yellow river in Shandong Jiqing Project scheduling operation plan analyses theory and practice. Theory and Practice of Operation of Interbasin Water Transfer Project. Paper Compilation on 2015 Chinese Hydraulic Engineering Annual Meeting: 799-803. 